\title{
High Thermal Stability and Flexibility of Thin Porous Ni Metal Support Prepared by Electroplating Deposition for Pd Alloy Membranes
}

\author{
Naruki Endo ${ }^{1, *}$, Satoshi Yaegashi ${ }^{2}$, Takayoshi Maehata ${ }^{2}$, Masashi Kumakawa ${ }^{1}$, Satoshi Suzuki ${ }^{1}$, \\ Kin-ichi Mashiko ${ }^{2}$ and Tetsuhiko Maeda ${ }^{1}$ \\ ${ }^{1}$ Renewable Energy Research Center, National Institute of Advanced Industrial Science and Technology (AIST), \\ Koriyama 963-0298, Japan \\ ${ }^{2}$ Sanno, Co. Ltd., Yokohama 223-0052, Japan
}

\begin{abstract}
A porous Ni film with numerous through-holes was prepared by electroplating deposition for use as a novel metal support for Pd alloy membranes. The porous Ni film with $5 \mu \mathrm{m}$ thickness maintained its shape after calcination in air at $500^{\circ} \mathrm{C}$ for $1 \mathrm{~h}$, which indicated its high thermal stability despite being electrodeposited. On the other hand, the calcined normal electrodeposited Ni crumbled and thus could not retain its original shape. The calcined porous Ni film also showed high mechanical strength, as its displacement was twice that of the normal Ni film. Hydrogen flow through the calcined porous Ni film was much higher than that observed for an approximately $5 \mu$ m-thick Pd alloy membrane, thus demonstrating the potential application of the film as a novel support for Pd alloy membranes in hydrogen production. [doi:10.2320/matertrans.M2017083]
\end{abstract}

(Received March 13, 2017; Accepted April 21, 2017; Published May 26, 2017)

Keywords: hydrogen separation, porous nickel metal support, electroplating deposition, palladium alloy membrane

\section{Introduction}

Hydrogen is one of the most promising candidates for energy carrier and storage medium. Hence, there has been increased interest in the production of pure hydrogen. Gas separation using membranes is a very attractive technology because it forms the basis of numerous chemical processes such as steam reforming of hydrocarbons. ${ }^{1)}$ Pd alloy membranes for hydrogen separation have been the focus of many studies owing to their potential application in hydrogen purification and/or membrane reactors. e.g. 2,3)

The common techniques for preparing Pd alloy membranes are sputter deposition, chemical vapor deposition (CVD), electroless plating (ELP) and electroplating deposition (EPD). ${ }^{4)}$ Although sputter deposition and CVD afford thin metal films at the nanoscale level, they require strictly regulated conditions and are thus not practically applicable. On the other hand, ELP is low cost and can allow for the plating of surfaces with any shape; hence, this is the most common preparation technique for Pd alloy membranes. ${ }^{4)}$ Numerous composite Pd alloy membranes prepared by ELP on ceramic supports have been reported. ${ }^{2-4)}$ However, the main drawbacks of ELP are the need for complicated pre-treatments and difficulties in controlling the film thickness.

EPD requires only simple equipment ${ }^{5)}$ and facilitates easy control of the film thickness by adjustment of the deposition time and/or current density; the other advantages are non-requirement of complicated pre-treatment, reproducibility, and easy large-lot production. The application of EPD, however, is limited to conducting support materials such as stainless steel. If a novel conducting support is developed, this method can be extended to the preparation of Pd alloy membranes.

Of late, we succeeded in using EPD for the preparation of a porous Ni film that shows high flexibility even after calcination in air. ${ }^{6)}$ Here, "porous" implies the presence of a large

*Corresponding author, E-mail: naruki.endo@aist.go.jp number of through-holes from a bottom to a surface of the film. Although, in general, the electrodeposited metal film after calcination shows poor flexibility owing to oxidation, our developed film shows better flexibility after calcination, indicating that it is stable under the conditions used for steam reforming of hydrocarbons. The porous Ni film has a comparable thermal expansion coefficient as that of Pd alloys and hence would be a suitable support for Pd alloy membranes; further, it allows for easy sealing in industrial assemblies and is low cost as compared to ceramic supports.

In the present study, we examined the crystal structure and surface texture of a porous $\mathrm{Ni}$ film, in addition to its thermal stability and flexibility. Furthermore, the hydrogen flow through the film was measured. These results were compared with those for a normal Ni film that was prepared by conventional EPD and had no through-holes. We demonstrate the potential applicability of the porous $\mathrm{Ni}$ film as a novel support for Pd alloy membranes.

\section{Experimental Procedure}

EPD was conducted using a counter electrode and a galvanostat. The deposition baths for porous $\mathrm{Ni}$ were prepared by dissolving the required amount of $\mathrm{NiCl}_{2}$ and an anionic surfactant (R1-O- $\left.\left(\mathrm{CH}_{2} \mathrm{CH}_{2} \mathrm{O}\right)_{n}-\mathrm{COOH} ; 12 \leq \mathrm{n} \leq 14\right)$ in a Watts bath. The normal Ni film for comparison was obtained by using an additive-free Watts bath. The main difference between preparation of porous/normal Ni films is that there is the anionic surfactant in the Watts bath or not. Ni films with thickness of 5-7 $\mu \mathrm{m}$ were electrodeposited on stainless steel substrates $(30 \mathrm{~mm} \times 30 \mathrm{~mm})$ under agitation. The deposition temperature, current density, and deposition time were in the range $40-65^{\circ} \mathrm{C}, 2-20 \mathrm{~A} / \mathrm{dm}^{2}$, and $5-10 \mathrm{~min}$, respectively. The electrodeposited Ni was easily delaminated from the substrate by cutting around the edge to obtain a $30 \mathrm{~mm} \times 30 \mathrm{~mm}$ film. The porous Ni film was obtained by calcination at $500^{\circ} \mathrm{C}$ for $1 \mathrm{~h}$ in air and subjected to acid treatment for several sec- 
onds for the removal of surface oxides.

The fabricated films were characterized by X-ray diffraction (XRD), scanning electron microscopy (SEM), and energy dispersive X-ray (EDX) spectrometry measurements. The XRD measurements were performed on a Rigaku Smartlab $\mathrm{X}$-ray diffractometer, using $\mathrm{Cu} K \alpha$ radiation. The scanned $2 \theta$ angles ranged from $20^{\circ}$ to $120^{\circ}$. The morphologies of the sample surface were observed by field emission scanning electron microscopy (FE-SEM; FEI QUANTA FEG250). Composition distribution was analyzed by an EDAX AMETEK OCTAN PRO EDX spectrometer.

Mechanical strength measurements for thin metal films $(\sim 5 \mu \mathrm{m})$ are generally difficult, because of which is no established method. Tensile tests are often used to evaluate the mechanical strength of electrodeposited films having various thicknesses; ${ }^{7,8)}$ however, these tests are limited to film thicknesses of $>20 \mu \mathrm{m}^{7)}$ as there is a risk of the film breaking during the test. We, therefore, propose an original method to evaluate the mechanical strength of the electrodeposited $\mathrm{Ni}$ film $(\sim 5 \mu \mathrm{m}){ }^{6}{ }^{6}$

Figure 1 (a) shows the instrument used for the mechanical strength measurements carried out on the as-prepared normal/porous Ni films, and calcined porous Ni film. These measurements were performed on a SHIMAZU EZ-TEST series EZ-SX system. Sample films of $20 \mathrm{~mm}$ diameter were cut from the $30 \mathrm{~mm} \times 30 \mathrm{~mm} \mathrm{Ni}$ film. The sample was held in place by a holder [Fig. 1 (b) bottom] and pressed by a spherical jig ( $\phi 7 \mathrm{~mm})$ [Fig. 1 (b) top]. The crosshead speed (CHS) was $1 \mathrm{~mm} / \mathrm{min}$. The calcined normal Ni film crumbled due to oxidation and hence could not be subjected to the measurements. For reference, the mechanical strength of rolled $\mathrm{Ni}$ films whose dimensions were the same as those of the electrodeposited films was evaluated before/after the calcination. Displacement against load force was measured within an error of $\pm 2 \%$.

Films with a diameter of $12 \mathrm{~mm}$ were also cut from the Ni film $(30 \mathrm{~mm} \times 30 \mathrm{~mm})$ for hydrogen flow measurements, which were performed by using a conventional gas-permeation apparatus. ${ }^{9,10)}$ The films were sealed by $\mathrm{Cu}$ gaskets (outside and inside diameter: $\phi 12.1 \mathrm{~mm} \times \phi 5.2 \mathrm{~mm}$ ), and then, hydrogen gas $(99.9999 \%)$ with a pressure of $100-189 \mathrm{kPa}$ and $100 \mathrm{kPa}$ was introduced on the upstream side $\left(P_{1}\right)$ and (a)

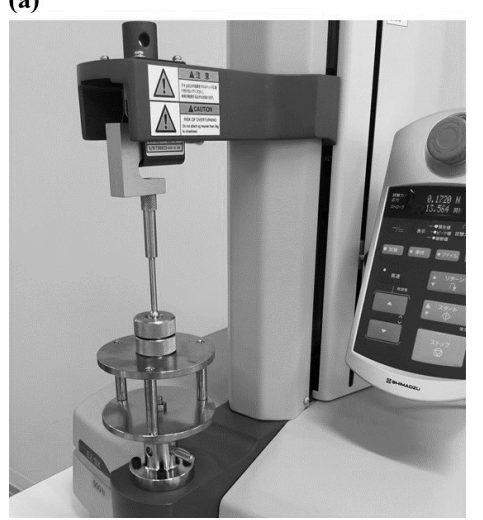

(b)

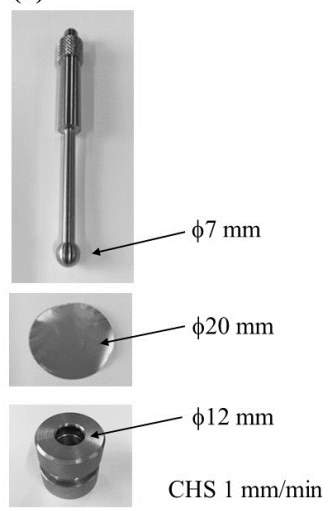

Fig. 1 Photographs of (a) mechanical strength measurement instrument and (b) spherical jig ( $\phi 7 \mathrm{~mm})$ (top), sample $(\phi 20 \mathrm{~mm})$ (middle,) and holder (bottom). The crosshead speed (CHS) is $1 \mathrm{~mm} / \mathrm{min}$. downstream side $\left(P_{2}\right)$, respectively. Hydrogen gas passing through the Ni film was measured by a mass flow meter (HORIBA MODEL SEF-E40; $20 \mathrm{~mL} / \mathrm{min}$ ) at $400^{\circ} \mathrm{C}$, where the Pd alloy membranes are usually operated.

\section{Result and Discussions}

Figure 2 shows the XRD patterns of normal/porous $\mathrm{Ni}$ films prepared by EPD. The XRD patterns of both normal and porous Ni before/after the calcination indicate an FCC structure, and there was no peak ( $2 \theta$ from $20^{\circ}$ to $120^{\circ}$ ) attributable to any other type of structure. The peaks due to both kinds of as-prepared films were broad because of the residual stress caused by EPD. The lattice constant calculated from each profile was good agreement with that of the Ni metal, ${ }^{11)}$ which indicates that the solution amount of the anionic surfactant in the crystal lattice of the as-prepared porous Ni was very low. As seen in the bottom part of Fig. 2 (b), the intensity of the 200 peak was higher than that of the 111 peak, implying that the as-prepared film showed preferential orientation along the (200) plane owing to the presence of the anionic surfactant during the film formation. However, the effect of the anionic surfactant on the growth direction is unclear at present. After the calcination [upper part of Fig. 2 (b)], the 111 peak became more prominent than the 200 peak owing to recrystallization. The XRD profiles of both calcined Ni films were sharp due to the annealing effect resulting from the calcination and were identical to each other.

Although the XRD patterns of calcined normal/porous $\mathrm{Ni}$ were the same, there was a significant difference in the thermal stability of the films after the air calcination. The insets in Figs. 2 (a) and (b) show the photographs of calcined normal/ porous $\mathrm{Ni}$, respectively. The normal film could not maintain its shape [Fig. 2 (a) inset] and was broken even by a very small contact force, i.e., which implied its poor flexibility. On the other hand, the porous $\mathrm{Ni}$ film maintained its shape and could be bent, i.e., it was highly flexible even after the calcination. Further, the porous Ni film had metallic luster because of the acid treatment carried out for the removal of surface oxides. There was no change in the porous Ni film after air calcination at $500^{\circ} \mathrm{C}$ for $1 \mathrm{~h}, 500^{\circ} \mathrm{C}$ for $24 \mathrm{~h}$ and $800^{\circ} \mathrm{C}$ for $1 \mathrm{~h}$, which confirmed its high thermal stability despite being electrodeposited.

The SEM images of the as-prepared/calcined porous $\mathrm{Ni}$ films are shown in Fig. 3 (a) and (b), respectively. We carried out SEM measurements on the calcined normal/porous Ni films after acid treatment. The SEM images of the normal Ni film are not shown here because those were almost similar to those of the as-prepared/calcined porous Ni film. As seen in Fig. 3 (a), there are many furrows on the film surface. On the other hand, for the surface texture of the calcined porous $\mathrm{Ni}$ film varied owing to the calcination, and there were many clear grain boundaries [see Fig. 3 (b)] because of the recrystallization texture formed by the calcination. Numerous micropores of $1-3 \mu \mathrm{m}$ could be seen in this film, as indicated by the open circles in the lower part of Fig. 3 (b). Such pores were not observed in the calcined normal Ni film.

The proposed mechanism underlying the formation of the micropores is as follows: The anionic surfactant used in the bath did not form a solid solution, but pillars of the condensed 
(a)

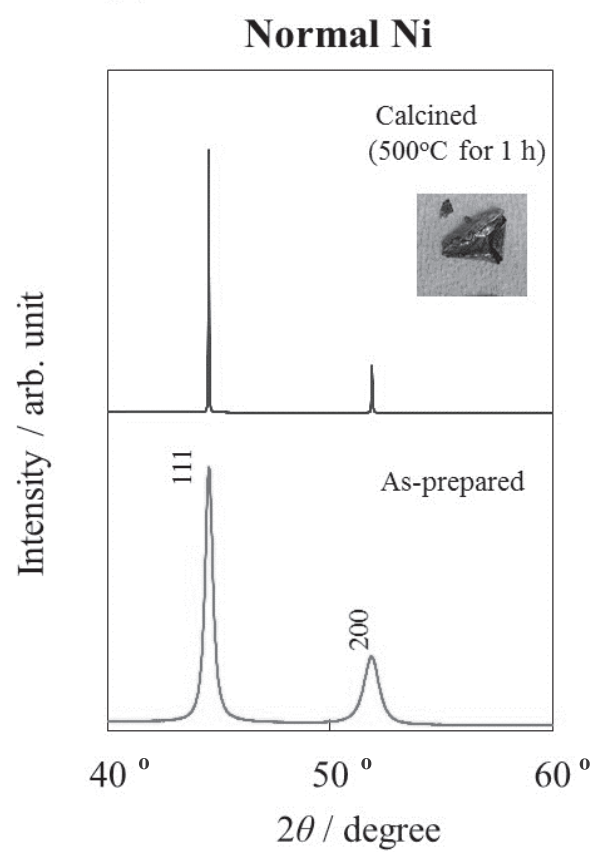

(b)

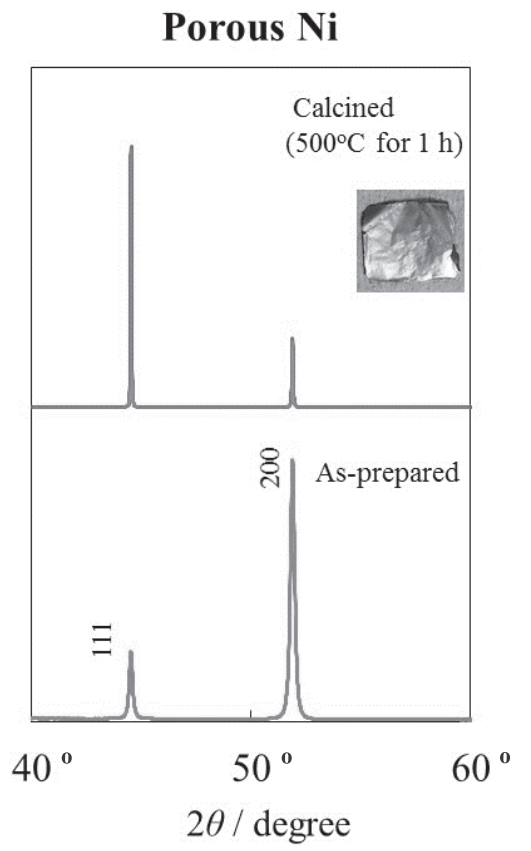

Fig. 2 XRD profiles of (a) normal Ni film before/after calcination and (b) porous Ni film before/after calcination. Insets are photographs of calcined normal $\mathrm{Ni}$ and calcined porous Ni films, respectively. The latter was subjected to acid treatment for surface oxide removal.

(a)
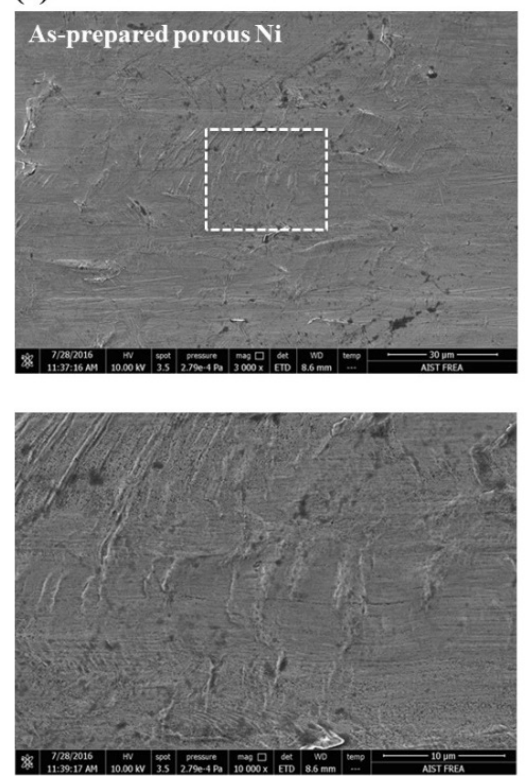

(b)
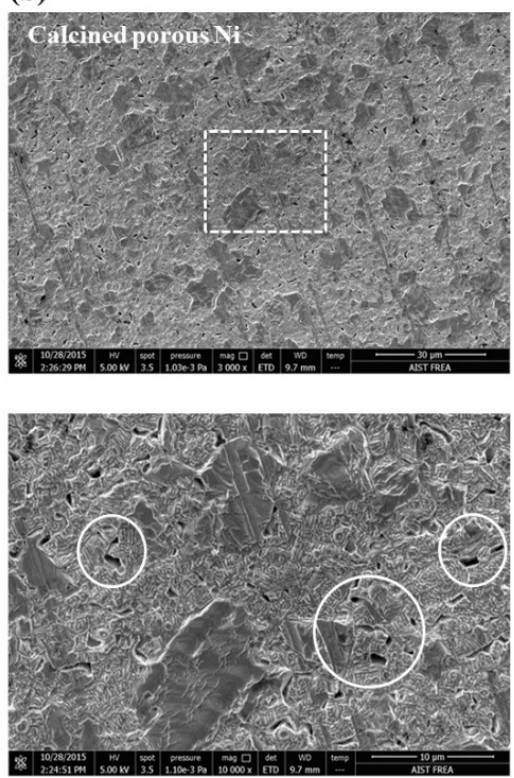

Fig. 3 SEM images of (a) as-prepared Ni and (b) calcined porous Ni films. Lower photographs are enlargements of upper ones indicated by dashed line squares. The calcined porous $\mathrm{Ni}$ film was subjected to acid treatment.

surfactant were selectively deposited during the film formation. Then, these pillars were burnt off by the air calcination to form micropores. Some of the micro pores would be through-holes that allow hydrogen gas to pass through the film.

Figure 4 presents the results of the mechanical strength measurements. The $y$-axis and $x$-axis depict the load force $(\mathrm{N})$ and displacement $(\mathrm{mm})$, respectively. The peak top indicates the break point of the film. The displacement of the porous film was higher than that of the normal film. Here, it was impossible to measure the mechanical strength of the calcined normal Ni film because it broke even when subjected to a very small contact force, as opposed to the case of the calcined porous Ni film. Despite lowering the peak top, the displacement was 1.7 times greater than that before the calcination, which indicates a unique mechanical property for the electrodeposited film. The displacements of the rolled $\mathrm{Ni}$ films before/after calcination were also measured for reference, and they were almost the same owing to work hardening by the rolling process. The displacements of the rolled films were less than $1 / 4$ of those for the calcined porous $\mathrm{Ni}$ film. 


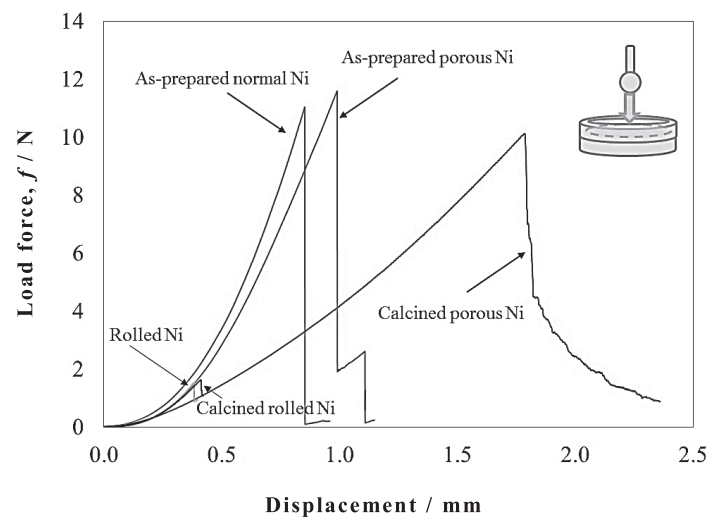

Fig. 4 Plots of load force, $f$, vs. displacement for as-prepared normal/porous $\mathrm{Ni}$, calcined porous $\mathrm{Ni}$, and rolled $\mathrm{Ni}$ films before/after calcination. The calcined normal Ni film could not be subjected to the measurements because it crumbled due to oxidation.

As described above, although the calcined normal/porous Ni films have the same crystal structure and similar surface textures, their mechanical properties are very different. The increasing displacement of the calcined porous $\mathrm{Ni}$ would be due to two reasons. First, oxidation suppression of the $\mathrm{Ni}$ metal occurred owing to the anionic surfactant existing in the as-prepared film. The anionic surfactant was selectively oxidized during the air calcination; therefore, the calcined film had a large unoxidized region and its metallic ductility was maintained-This is the reason for the high stability of the porous Ni film during the calcination. Secondly, the pores formed after the calcination facilitated strain release during the mechanical strength measurements. As a result, the peak top decreased but the displacement increased, i.e., the calcined porous Ni had high flexibility.

Figure 5 shows the rates of hydrogen flow through the electrodeposited $\mathrm{Ni}$ films at $400^{\circ} \mathrm{C}$. The hydrogen flow rates were only measured for the calcined porous Ni film. The hydrogen flow rates were found to be proportional to the pressure difference, $\Delta P\left(=P_{1}-P_{2}\right)$, which implied that some of the formed pores in the film were through-holes. In fact, the formation of through-holes has been described elsewhere. ${ }^{12,13)}$ The maximum hydrogen flow rate was limited to $20 \mathrm{~mL} / \mathrm{min}$ by the mass flow controller (MFC) on the upstream side, so that any higher hydrogen flow rate would be measured if an MFC with a high flow range was used.

Finally, SEM observations carried out after the hydrogen flow measurements confirmed that the calcined film was not broken and that there were no micro-cracks and pinholes on the film. Note that the hydrogen flow at each $\Delta P$ was much higher than that observed when using $\sim 5 \mu$ m-thick Pd alloy membranes. In other words, the calcined porous Ni would not obstruct the passage of hydrogen gas through the Pd alloy membranes and thus would act as a suitable membrane support. Further studies are underway to prepare a composite Pd alloy/porous Ni membrane by EPD.

\section{Conclusions}

A porous Ni film of $5 \mu \mathrm{m}$ thickness was prepared by electroplating deposition (EPD), and its thermal stability and mechanical strength were evaluated. The porous film maintained

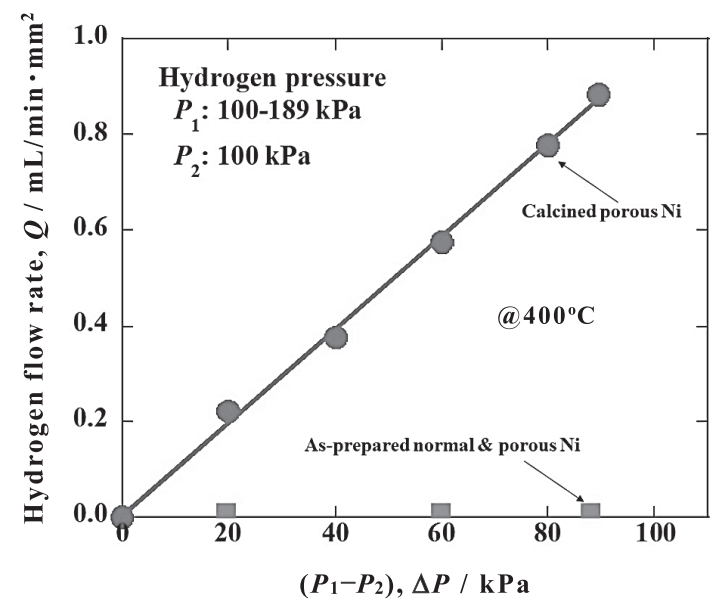

Fig. 5 Plots of hydrogen flow through the film, $Q$, vs. pressure difference, $\Delta P,(0-89 \mathrm{kPa})$ for as-prepared normal/porous $\mathrm{Ni}$ and calcined porous $\mathrm{Ni}$ films at $400^{\circ} \mathrm{C}$. No hydrogen flow was observed in both as-prepared films.

its shape after the air calcination, which indicated its high thermal stability despite being electrodeposited. The calcined porous Ni film showed superior flexibility, as opposed to the as-prepared normal/porous Ni film. High hydrogen flow rates through the calcined porous Ni film were observed, which indicated that the porous Ni film prepared by EPD would be a novel metal support for Pd alloy membranes.

\section{Acknowledgments}

This work was supported by the Seeds Support Program for companies in the disaster region of the Great East Japan Earthquake, sponsored by the Fukushima Renewable Energy Institute, AIST (FREA). The authors would like to acknowledge Dr. Kosone for the XRD measurements and Dr Nishimura for the hydrogen flow measurements.

\section{REFERENCES}

1) J. Shu, B.P.A. Grandjean, A. Van Neste and S. Kaliaguine: Can. J. Chem. Eng. 69 (1991) 1036-1060.

2) F. Gallucci, E. Fernandez, P. Corengia and M. van Sint. Annaland: Chem. Eng. Sci. 92 (2013) 40-66.

3) R. Dittmeyer, V. Höllein and K. Daub: J. Mol. Catal. Chem. 173 (2001) 135-184.

4) S. Yun and S.T. Oyama: J. Membr. Sci. 375 (2011) 28-45.

5) S.E. Nam, S.H. Lee and K.H. Lee: J. Membr. Sci. 153 (1999) 163-173.

6) N. Endo, T. Kosone, S. Suzuki, M. Kumakawa, T. Maeda, S. Yaegashi, T. Watanabe and K. Mashiko: Proc. of $35^{\text {th }}$ Meeting of Hydrogen Energy System Society of Japan, Tokyo (2015) pp.109-110.

7) J. Lou, P. Shrotriya, S. Allameh, T. Buchheit and W.O. Soboyejo: Mater. Sci. Eng. A 441 (2006) 299-307.

8) Y. Yang, N. Yao, W.O. Soboyejo and C. Tarquinio: Scr. Mater. 58 (2008) 1062-1065.

9) C. Nishimura, M. Komaki and M. Amano: Mater. Trans., JIM 32 (1991) 501-507.

10) M. Amano, M. Komaki and C. Nishimura: J. Less Common Met. 172174 B (1991) 727-731.

11) M. Ellner, K. Kolatschek and B. Predel: J. Less Common Met. 170 (1991) 171-184.

12) S. Yaegashi, T. Watanabe, Y. Kato, H. Hatsuda, J. Ryu: Japan Patent JP2016-094659A.

13) S. Yaegashi, T. Watanabe, Y. Kato, H. Hatsuda, J. Ryu: Japan Patent JP2017-039083A. 